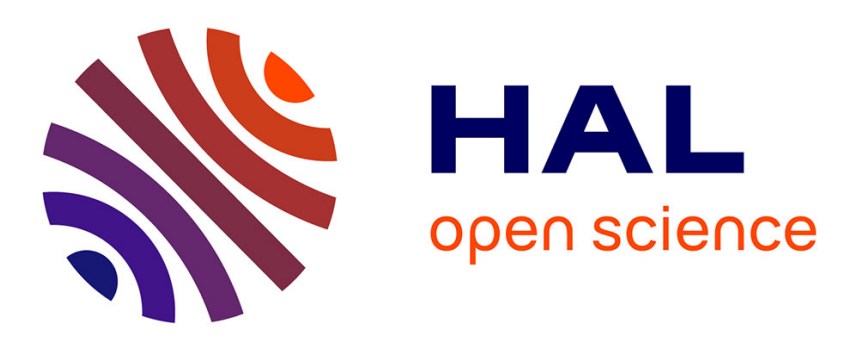

\title{
Adaptive attitude control of a microsatellite during payload deployment
}

Harmonie Leduc, Christelle Pittet, Dimitri Peaucelle

\section{To cite this version:}

Harmonie Leduc, Christelle Pittet, Dimitri Peaucelle. Adaptive attitude control of a microsatellite during payload deployment. 2017. hal-01473674

\section{HAL Id: hal-01473674 \\ https://hal.science/hal-01473674}

Preprint submitted on 22 Feb 2017

HAL is a multi-disciplinary open access archive for the deposit and dissemination of scientific research documents, whether they are published or not. The documents may come from teaching and research institutions in France or abroad, or from public or private research centers.
L'archive ouverte pluridisciplinaire HAL, est destinée au dépôt et à la diffusion de documents scientifiques de niveau recherche, publiés ou non, émanant des établissements d'enseignement et de recherche français ou étrangers, des laboratoires publics ou privés. 


\section{Adaptive attitude control of a microsatellite during payload deployment}

\author{
Harmonie Leduc \\ hleduc@laas.fr
}

\author{
Christelle Pittet \\ christelle.pittet@cnes.fr
}

\author{
Dimitri Peaucelle \\ peaucell@laas.fr
}

\begin{abstract}
A robust direct adaptive attitude law is implemented in a attitude control system simulator. The meaning of robustness here is with respect to uncertainties in the inertia of the satellite. The controller has already been proved to be more robust than a corresponding robustly stabilizing static output feedback. The aim of the study is to show that in practice as well, only the adaptive controller can stabilize the satellite whatever the number of deployed/folded payload masts, namely whatever the value of its inertia.
\end{abstract}

\section{Introduction}

Attitude control of satellites has been a rising issue for years [1], [19], [11], [24]. Regarding the evolution of the missions, more and more accurate attitude controllers are required. The AOCS control law in mission mode currently implemented on CNES microsatellites consists in a switching controller [17]: A angular rate controller allows a fast but non accurate reduction of the depointing until it reaches a switching value. Below it, a proportional-derivative (PD) law aims at controlling precisely a small attitude error. This commutative controller turns out to be efficient but in practice, any change or uncertainty in a parameter of the satellite forces the controller to be redesigned in order to be compliant to reaction wheel speed limited angular momentum.

To solve this problem, the idea of computing a time varying controller, updated thanks to the measurements, has been proposed both in theory
[21], [20], [3] and in practice [23], [14], [25], [4]. At CNES, such a direct adaptive controller has been designed [12] and successfully tested in flight [18]. However, the robustness of such a controller with respect to changes in the satellite inertia has not been fully studied. This issue is most relevant in practice: The deployment of the payload masts of the satellite involves important changes of its inertia.

This topic has been considered theoretically in [8] and [9] and applied to a simple three axes linear model in [7]. The gains of the designed adaptive controller are proved to be bounded and evolve around a known stabilizing baseline ([6], [16], [15]). The goal of this study is to apply these theoretical improvements to a complex AOCS simulator where deployment/folding scenarii can be simulated. The aim is to illustrate that as in theory, the adaptive controller allows to stabilize the satellite for all possible values of its inertia.

The paper is organized as follows: The current satellite attitude controller is presented in section II. In section III, important theoretical results about the design of the robust adaptive controller are recalled. Then, the results obtained with the CNES AOCS simulator are given in section IV, and compared with the ones obtained with a static controller baseline. Conclusion and outlooks for future works are given in section V. 


\section{Switching control law of Myriade satellites in normal mode}

CNES microsatellite productline (Myriade) AOCS architecture is based on 3-axis stabilization and consists in four modes: The Acquisition and Safehold Mode (MAS) is used after separation from the launcher and later in case of anomaly detection, and aims at pointing the spacecraft towards the Sun. The coarse pointing mode (MGT) is a transition mode from acquisition to normal mode, based on magnetic stabilization. The Orbit Control Mode (MCO) is dedicated to perform the orbit maneuvers.

The Normal Mode (MNO) is the mode in which the scientific mission is carried out. The spacecraft is 3-axis stabilized by three reaction wheels (one on each axis). Attitude is only given by the star sensor, which imposes some strategy to cope with possible drops of measurement. In MGT and MNO modes, the guidance profile is given by sending commands either as harmonic series or as polynomial functions. During the normal mode, the magneto-torquer bars unload the angular momentum of the wheels.

MNO reaction wheels control law has been detailed in several former papers [17], [22], [13], and will only be reminded here. It consists in a switching law, with a proportional-derivative form at low depointing, and a 'speed bias' control at higher depointing such as:

- If $\left|e_{\theta}\right|>\theta_{t h}$,

$$
T_{a}=-K\left(\omega_{e}+\operatorname{sign}(\theta) \Omega_{b}\right)
$$

- If $\left|e_{\theta}\right| \leq \theta_{t h}$

$$
T_{a}=-K_{r \theta} e_{\theta}-K_{r \omega} \omega_{e}
$$

where $e_{\theta}=\theta-\theta_{r}$ is the 3 -axis estimated pointing error, $\omega_{e}$ the 3 -axis estimated angular rate error, $\theta_{t h}$ is the 3 -axis depointing error threshold, $\Omega_{b}$ is the 3 -axis bias angular rate, $K, K_{r \theta}$ and $K_{r \omega}$ are 3 -axis tuning gains, and $T_{a}$ is the 3 -axis switching torque.
The switching law is followed by a LTI stabilizing filter $F(s)$ of PID type, such that $T_{p}=F(s) T_{a}$, with $T_{p}$ the 3 -axis reaction wheel control input in satellite frame. This switching control law is nonlinear and time varying and already depends on the depointing value.

Many difficulties are linked to this control law: The first one is the torque continuity which has to be ensured at the switching point $\theta_{t h}$, to avoid pointing error transient. For that purpose, the tuning parameters of the two switching laws are linked, so that when $\left|e_{\theta}\right|=\theta_{t h}$, both switching torques are equal. In practice, at the switching point, a discontinuity remains: Indeed, because of the sampling time of the AOCS software and measurement noise of star sensor, one never gets exactly $\left|e_{\theta}\right|=\theta_{t h}$.

The second problem is the choice of the angular rate bias value, which has to be consistent with the limited reaction wheel angular momentum and momentum dumping capacity by magneto-torquers (which strongly depends on the day and orbital position).

Because of that, the stability assessment of the switching closed loop needs exhaustive simulation campaigns to cover the whole pointing error domain. For these reasons, we have decided to search for a new control law which could take into account high or low pointing errors without torque discontinuity, which would be consistent with reaction wheels capacity limits, and with a priori stability proof over the pointing error domain.

\section{Robust reaction wheel adap- tive control law}

\subsection{Luzi's adaptive controller}

During his $\mathrm{PhD}$ [13], Luzi proposed to replace the switching controller part by the time varying Proportional-Derivative controller $T_{a}(t)=K(t) e(t)$, followed by the existing stabilizing filter $F(s)$, where $e(t)^{T}=\left[e_{\theta}(t) \omega_{e}(t)\right]$ is the controller input and $K(t)=\left[K_{\theta}(t) K_{\omega}(t)\right]$ are the adaptive gains.

Thus, if the time varying $\mathrm{PD}$ gains tend to the PD switching gains at low pointing error, the pointing performances will be kept for the satellite. The new 


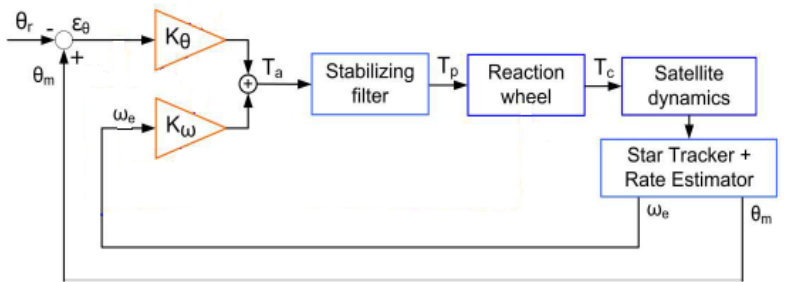

Figure 1: Reaction wheel control loop with Luzi adaptive controller

closed-loop structure is illustrated in Figure 1, with $K_{\theta}$ and $K_{\omega}$ the adaptive gains, $\omega_{r}$ the reaction wheel angular rate, $\theta_{r}$ the pointing reference, $\theta_{m}$ the pointing estimate, and $T_{c}$ the torque applied to the satellite by the reaction wheels.

Adaptive gains $K_{\theta}$ and $K_{\omega}$ are time varying, and the parameters which determine their variation law are computed by solving LMIs (Linear Matrix Inequalities) (see [12] for details). When computing these adaptive parameters, all the parameters of the satellite model are supposed perfectly known. Yet, in practice, this is not the case. Hence, some modifications have been brought to Luzi adaptive controller to make it more robust with respect to model parameter uncertainties [8], [9].

In all the following, it is supposed that the inertia of the satellite $J$ is subject to uncertainties. Only its nominal value $J_{\text {nom }}$ is known, and we aim at designing a controller which would stabilize the satellite for the largest interval of inertia.

\subsection{Robust adaptive controller design}

In [8] and [9], Luzi adaptive controller has been improved in order to fit better with parametric uncertainties. Let us consider the following time varying controller:

$$
T_{a}(t)=K(t) e(t)
$$

where $e(t)^{T}=\left[e_{\theta}(t) \omega_{e}(t)\right]$ is the controller input and $K(t)=\left[K_{\theta}(t) K_{\omega}(t)\right]$, as in [13]. Here, the controller gains are solutions of the following differential equations:

$$
\begin{aligned}
& K_{\theta_{k}}(t)=\mathcal{I}_{D_{\theta_{k}}}\left(\dot{K}_{\theta_{k}}(t), W_{\theta_{k}}(t)\right) \\
& W_{\theta_{k}}(t)=\gamma_{\theta_{k}}\left(-G_{\theta_{k}} e(t) e_{\theta_{k}}(t)-\sigma_{\theta_{k}}\left(K_{\theta_{k}}(t)-K_{r \theta_{k}}\right)\right)
\end{aligned}
$$

and

$$
\begin{aligned}
& K_{\omega_{k}}(t)=\mathcal{I}_{D_{\omega_{k}}}\left(\dot{K}_{\omega_{k}}(t), W_{\omega_{k}}(t)\right) \\
& W_{\omega_{k}}(t)=\gamma_{\omega_{k}}\left(-G_{\omega_{k}} e(t) e_{\omega_{k}}(t)-\sigma_{\omega_{k}}\left(K_{\omega_{k}}(t)-K_{r \omega_{k}}\right)\right)
\end{aligned}
$$

where $k \in\{x, y, z\}$ designs the satellite axes. The values of $G_{\theta_{k}}$ and $G_{\omega_{k}}$ give the direction of the adaptation. $D_{\theta_{k}}\left(D_{\omega_{k}}\right.$ respectively) enters in the definition of the saturated integrator $\mathcal{I}_{D_{\theta_{k}}}\left(\mathcal{I}_{D_{\omega_{k}}}\right.$ respectively), which pushes $K_{\theta_{k}}(t)$ ( $K_{\omega_{k}}(t)$ respectively) inside a set $\mathcal{E}_{\theta_{k}}\left(\mathcal{E}_{\omega_{k}}\right.$ respectively) when it is at its border, so that the adaptive gains are bounded. The sets $\mathcal{E}_{\theta_{k}}$ and $\mathcal{E}_{\omega_{k}}$ are defined by:

$K_{\theta_{k}}$ $\in \mathcal{E}_{\theta_{k}}$

$\Leftrightarrow \operatorname{Tr}\left(\left(K_{\theta_{k}}(t)-K_{r \theta_{k}}\right)^{T} D_{\theta_{k}}\left(K_{\theta_{k}}(t)-K_{r \theta_{k}}\right)\right) \leq 1$

and

$$
\begin{aligned}
& K_{\omega_{k}} \in \mathcal{E}_{\omega_{k}} \\
& \Leftrightarrow \operatorname{Tr}\left(\left(K_{\omega_{k}}(t)-K_{r \omega_{k}}\right)^{T} D_{\omega_{k}}\left(K_{\omega_{k}}(t)-K_{r \omega_{k}}\right)\right) \leq 1
\end{aligned}
$$

where $\operatorname{Tr}$ is the trace operator.

The forgetting factors $\sigma_{\theta_{k}}$ and $\sigma_{\omega_{k}}$ drive $K_{\theta_{k}}(t)$ and $K_{\omega_{k}}(t)$ to $K_{r \theta_{k}}$ and $K_{r \omega_{k}}$ when $e(t)$ is zero ([10], [2], [5]). The value of $\gamma_{k}$ gives the variation rate of $K_{k}(t)$. Theorem 3.1 provides a method to design an adaptive controller which is proved to be more robust than its baseline.

Theorem 3.1 If $K_{a}(t)=\left[K_{r \theta} K_{r \omega}\right]$ e(t) robustly stabilizes the satellite, then there exist matrices $P^{\left[v_{J}\right]} \succ 0, S, G=\left[G_{\theta_{x}}^{T}, \ldots, G_{\omega_{x}}^{T}, \ldots\right]^{T}$ and $D=$ $\operatorname{diag}\left(D_{\theta_{x}}, \ldots, D_{\omega_{x}}, \ldots\right)$ such that, for all $v_{J}$, 


$$
\mathcal{L}\left(K_{r \theta}, K_{r \omega}, P^{\left[v_{J}\right]}, S, G, D\right) \prec 0
$$

where $v_{J}$ means that the value of the matrix $P^{\left[v_{J}\right]}$ can be different from one tested value of the inertia $J$ to another.

Consider now the following bilinear matrix inequalities (BMIs) for all $v_{J}$, with $\breve{P}^{\left[v_{J}\right]} \succ 0$ :

$$
\mathcal{B}\left(\breve{K}_{0}, \breve{P}^{\left[v_{J}\right]}, \breve{G}, \breve{D}, \breve{\epsilon}, \breve{F}\right) \prec 0 .
$$

These constraints are such that:

- If we fix $\breve{K}_{0}=\left[\begin{array}{ll}K_{r \theta} & K_{r \omega}\end{array}\right], \breve{G}=G$ and $\breve{D}=D$ $G_{\theta_{k}}, G_{\omega_{k}}, D_{\theta_{k}}$ and $D_{\omega_{k}}$, BMIs of Theorem 3.1 become LMIs and then are solvable with classical solvers.

- For such values of $\breve{K}_{0}, \breve{G}$ and $\breve{D}$, the LMIs of Theorem 3.1 are feasible.

- If the constraints are feasible for the extremal values of the uncertain vector (or in that case for the extremal values of $J$ ), then $\breve{F}$ is such that $K_{a}(t)=\left(\breve{K}_{0}+\breve{F}+K(t)\right) e(t)$ stabilizes the satellite for any value of the inertia $J$.

- If the constraints are feasible for the extremal values of $J$, then whatever positive $\gamma_{\theta_{k}}$ and $\gamma_{\omega_{k}}$ the new adaptive control robustly stabilizes the satellite when all $\sigma_{\theta_{k}}$ and $\sigma_{\omega_{k}}$ are zero and robustly stabilizes a neighborhood of the target attitude and target angular speed set when at least one $\sigma_{\theta_{k}}$ or $\sigma_{\omega_{k}}$ is strictly positive.

Expressions of (8) and (9) and a detailed proof of Theorem 3.1 are given in [9].

\subsection{Implementation in discrete time}

The adaptive control law is discretized to be implemented in the AOCS software. For that purpose, the Euler transformation has been chosen. The discretetime expression of the control law is given by:

$$
\begin{aligned}
K_{\theta_{k}}(n) & =K_{\theta_{k}}(n-1)-\gamma_{\theta_{k}}\left(G_{\theta_{k}} e(n-1) e_{\theta_{k}}(n-1)\right. \\
& \left.+\sigma_{\theta_{k}} K_{\theta_{k}}(n-1)\right)
\end{aligned}
$$

$$
\begin{aligned}
K_{\omega_{k}}(n) & =K_{\omega_{k}}(n-1)-\gamma_{\omega_{k}}\left(G_{\omega_{k}} e(n-1) e_{\omega_{k}}(n-1)\right. \\
& \left.+\sigma_{\omega_{k}} K_{\omega_{k}}(n-1)\right)
\end{aligned}
$$

In (10) and (11), indices $n$ and $n-1$ stand for current and past instant respectively. The boundedness of the gains is obtained by implementing the following procedures:

$$
\begin{aligned}
& \text { - } \text { if } K_{\theta}(n)>K_{\theta_{\max }} \text {, then } K_{\theta}(n)=K_{\theta_{\max }} \\
&- \text { else if } K_{\theta}(n)<K_{\theta_{\min }} \text {, then } K_{\theta}(n)=K_{\theta_{\min }} \\
& \text { - } \quad \text { - if } K_{\omega}(n)>K_{\omega_{\max }}, \text { then } K_{\omega}(n)=K_{\omega_{\max }} \\
&- \text { else if } K_{\omega}(n)<K_{\omega_{\min }}, \quad \text { then } \\
& K_{\omega}(n)=K_{\omega_{\min }}
\end{aligned}
$$

\section{Validation}

Launched in 2004, DEMETER was the first satellite of the MYRIADE series. It has observed electric and magnetic signals in Earth's ionosphere for more than six years. Collected data have allowed to answer questions about the effect of seismic and volcanic activity to the electromagnetic environment of Earth's atmosphere. Its orbit was near-sun-synchronous with $660 \mathrm{~km}$ altitude.

In practice, the inertia of a satellite can be subject to important variations, for example during the deployment/folding of the payload masts (see Figure 2). Luzi's controller, introduced in section III, has been designed to fitted well with nominal inertia. In all the following, the nominal inertia $J_{d e p}$ of the satellite is considered equal to the inertia of the satellite when all the masts are deployed, and $J_{\text {fold }}$ denotes the inertia of the satellite when all the panels are folded. For DEMETER satellite, $J_{\text {fold }}$ is equal to $J_{\text {dep }}$ reduced by $70 \%$ (i.e. $J_{\text {fold }}=0,3 J_{\text {dep }}$ ). 


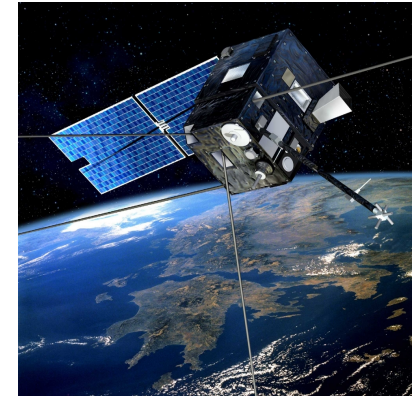

Figure 2: DEMETER artist view

\subsection{Theoretical results}

In [9], parameters $G_{\theta_{k}}, G_{\omega_{k}}, D_{\theta_{k}}, D_{\omega_{k}}, \sigma_{\theta_{k}}, \sigma_{\omega_{k}}$, $\gamma_{\theta_{k}}$ and $\gamma_{\omega_{k}}$ of the adaptive controller (3) have been computed by solving LMIs (8).

Then, Theorem 3.1 has been used as follows: On one hand, the value of $\breve{K}_{0}$ is supposed to be the one of the static output feedback $\left[\begin{array}{ll}K_{r \theta} & K_{r \omega}\end{array}\right]$. It is then shown that the satellite model controlled by this static output feedback but with inertia $J$ considered uncertain at $\pm 70 \%$ (so that $J_{\text {fold }}$ is in the interval of uncertainty) is not stable. More precisely, it is shown that with this static output feedback, the satellite is robustly stable with inertia $J$ considered uncertain at $\pm 33 \%$ ( $J_{\text {fold }}$ is not in this interval of uncertainty), but not $\pm 34 \%$. On the other hand, the values of parameters $G_{\theta_{k}}, G_{\omega_{k}}, D_{\theta_{k}}, D_{\omega_{k}}, \sigma_{\theta_{k}}, \sigma_{\omega_{k}}, \gamma_{\theta_{k}}$ and $\gamma_{\omega_{k}}$ are saved and put in BMIs (9), considering that inertia $J$ is uncertain at $\pm 70 \%$ (so that $J_{\text {fold }}$ is in the interval of uncertainty). Constraints (9) of Theorem 3.1 turn out to be feasible, which gives a value for the adaptive parameter $\breve{F}$ and then a "new" adaptive controller $K_{a}(t)=\left(\breve{K}_{0}+K(t)+\breve{F}\right) e(t)$. More precisely, it is shown that the limit of feasibility of the constraints of Theorem 3.1 is reached when $J$ has $\pm 89 \%$ of uncertainty. Details about the application of Theorem 3.1 can be found in [9].

These results are summed up in Table 1.
Table 1: Maximal Uncertainty On The Inertia For Which The Satellite Can Be Stabilized

\begin{tabular}{|l|l|l|}
\hline & limit & {$\left[J_{\text {fold }} ; J_{\text {dep }}\right]$ included } \\
\hline Static & $33 \%$ & no \\
\hline Adaptive & $89 \%$ & yes \\
\hline
\end{tabular}

\subsection{Validation on AOCS simulator}

The new control algorithm has been tested with the same validation process as any operational AOCS software. The new control law has been validated on DEMETER AOCS simulator. This validation was based on a simulation campaign to assess performances and robustness of this controller with respect to a large amount of contributors. Two types of controllers have been tested: A static one $K_{a}(t)=\left[\begin{array}{ll}K_{r \theta} & K_{r \omega}\end{array}\right] e(t)$ and an adaptive $K_{a}(t)=\left(\breve{K}_{0}+\breve{F}+K(t)\right) e(t)$. Notice that the static controller corresponds to the switching controller where the switching threshold has been increased in order to avoid to switch to speed bias configuration.

The results presented in this subsection deal with deployment/folding scenarii. In Figures 4 and 5, the following scenario has been simulated:

- From $t=0$ to $t=100 \mathrm{~s}$, the satellite masts are all folded.

- At $t=100 \mathrm{~s}$, the 4 th panel is deployed.

- At $t=200 \mathrm{~s}$, the 5 th panel is deployed.

- At $t=300 \mathrm{~s}$, the 1 th panel is deployed.

- At $t=400 \mathrm{~s}$, the 2 th panel is deployed.

- At $t=500 \mathrm{~s}$, the 3 th panel is deployed, so that between $t=500$ and $t=600 \mathrm{~s}$, all the panels are deployed.

This means that along the simulations, the inertia $J$ of the satellite varies from $J_{\text {fold }}$ to $J_{d e p}$. Considering the results obtained in the previous subsection, only the adaptive controller is expected to 

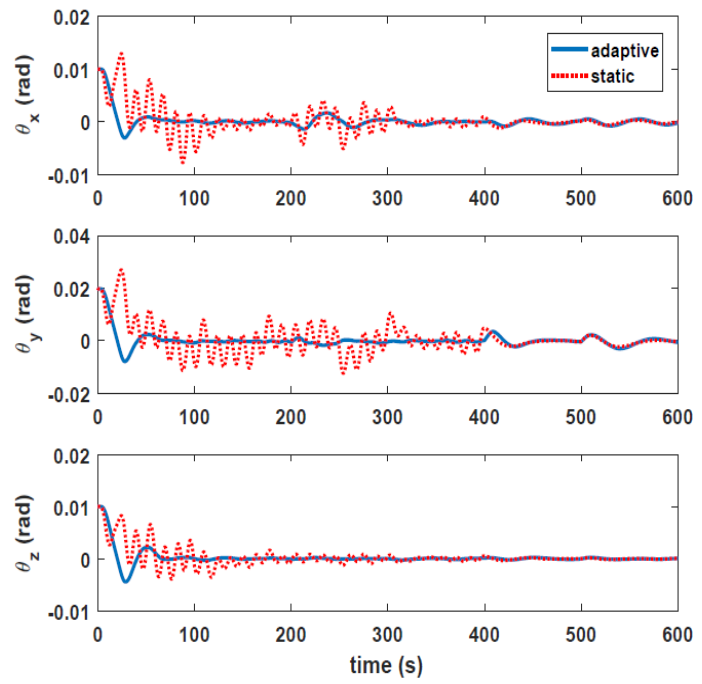

Figure 3: Attitude angle without wheel torque saturation. Red dashed: Static; Blue solid: Adaptive
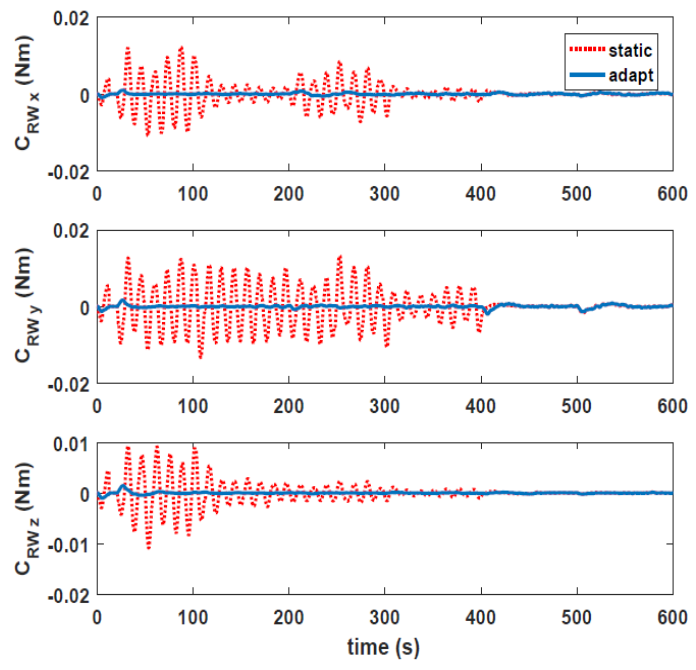

Figure 4: Reaction wheel torque before wheel torque saturation. Red dashed: Static; Blue solid: Adaptive stabilize the satellite whatever the value of its inertia.

Figures 3 and 4 show the time evolutions of attitude angle $\theta$ and reaction wheel torque $C_{R W}$ respectively, where the reaction wheel torque saturates if exceeding $0,005 \mathrm{Nm}$ (which is the case in practice). In Figure 5, the red dashed lines are for static controller and the blue solid lines are for the adaptive controller.

Considering Figures 3 and 4 , it is clear that the adaptive controller achieves a better performance than the static one in terms of robustness with respect to changes of inertia. Solid blue curves in Figure 4 attest to the fact that the adaptation makes the reaction wheel operate less. Without adaptation (red dashed curves), the reaction wheel torque have some important variations.

However, it has been shown in [9] and recalled in the last subsection that the static controller is not supposed to stabilize the satellite for all the inertia between $J_{\text {fold }}$ and $J_{\text {dep }}$. Yet, Figures 3 and 4 seem to show that the satellite is stable with the static controller. Actually, the saturation of the reaction wheel torque prevents the satellite to turn unstable. Of course this saturation was not taken into account in the linear modelisation of the satellite in section III. The role of the wheel torque saturation is proved in Figure 5, which shows the time variations of attitude angle $\theta$ for the same scenario as upper, but without considering the possible saturation of the reaction wheel torque. The solid blue curves on the right of Figure 5 are also plotted separately with a different scale on the left for better visibility. Dashed red curves show that the satellite is not stable with the static controller in that case, whereas the adaptive controller still achieves a good performance. This simulation confirms that the adaptive controller is more robust than the static controller.

Now, let us consider the $5 !=120$ possible deployment/folding scenarii. The reaction wheel torque saturation is again taken into account. For all 120 scenarii, the maximal depointing, angular speed and reaction wheel speed after $n=0,100, \ldots, 500$ s of 

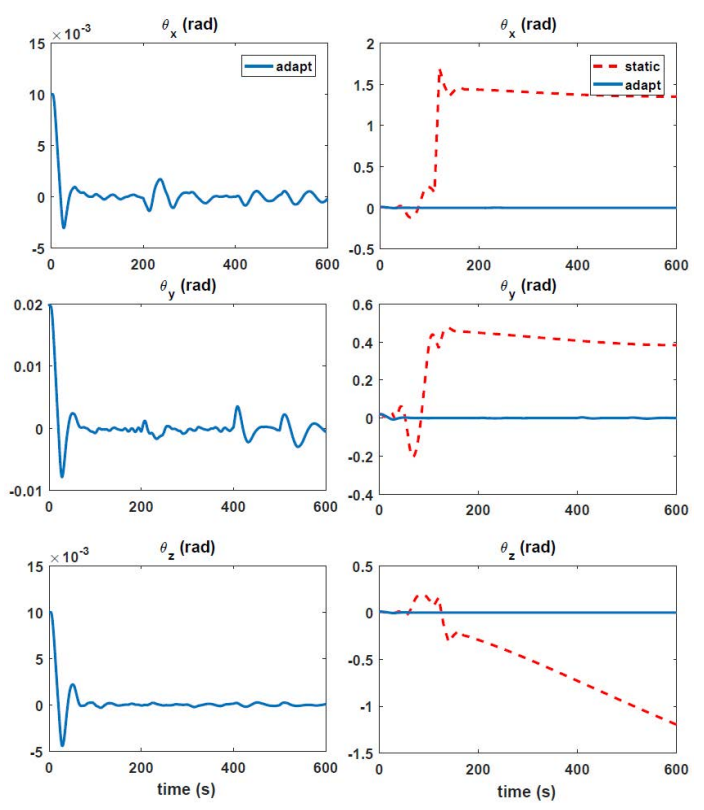

Figure 5: Attitude angle with wheel torque saturation. Red dashed: Static; Blue solid: Adaptive

simulation have been computed. For example, the maximal depointing after 200s is the maximal absolute value of the attitude angles measured between 200s and the end of the simulation. Table 2 gives the mean of this values on the 120 scenarii for static and adaptive controller and for $x$-axis. These mean values show that what holds for the "45123" scenario analyzed upper holds for the 120 scenarii as well: both static and adaptive controller allow to stabilize the satellite (thanks to the wheel torque saturation for the static controller), but the stabilization of the satellite is much faster and smoother with the adaptive controller.

\section{Conclusion}

The robust adaptive attitude controller designed in [9] has been applied to an AOCS simulator. Theoretically, the adaptive controller is supposed to stabilize the robust whatever the value of its inertia, whereas the corresponding static controller can only
Table 2: Maximal depointing (deg), angular speed $(\mathrm{deg} / \mathrm{s})$ and wheel speed $(\mathrm{deg} / \mathrm{s})$ after $\mathrm{n}$ seconds: mean on 120 scenarii

\begin{tabular}{|l|l|l|l|l|l|l|}
\hline & \multicolumn{2}{|c|}{$\theta$} & \multicolumn{2}{c|}{$\omega$} & \multicolumn{2}{c|}{$\omega_{r}$} \\
\hline $\mathrm{n}$ & static & adapt & static & adapt & static & adapt \\
\hline \hline 0 & 0,652 & 0,305 & 0,033 & 0,018 & 119 & 904 \\
\hline 100 & 0,401 & 0,075 & 0,020 & 0,002 & 777 & 527 \\
\hline 200 & 0,220 & 0,067 & 0,010 & 0,002 & 571 & 474 \\
\hline 300 & 0,065 & 0,057 & 0,003 & 0,001 & 470 & 471 \\
\hline 400 & 0,042 & 0,048 & 0,001 & 0,001 & 456 & 467 \\
\hline 500 & 0,027 & 0,034 & 0,001 & 0,001 & 445 & 456 \\
\hline
\end{tabular}

stabilize the satellite for a twice smaller range of inertia. In practice, simulations show that the adaptive controller robustly stabilizes the satellite whatever the number of deployed/folded masts (all masts deployed standing for the highest inertia, all masts folded for the smallest). The next step of the study will be to implement the adaptive controller on an avionic bench.

\section{References}

[1] O. Egeland and J.-M. Godhavn. Passivity based adaptive attitude control of a rigid spacecraft. IEEE Transactions on Automatic Control, 39(4):842-846, 1994.

[2] V. Fomin, A. Fradkov, and V. Yakubovich. Adaptive control of dynamic plants. Nauka, Moscow, 1981. In Russian.

[3] A.L. Fradkov, D. Peaucelle, and B.R. Andrievsky. Robust adaptive passification and stabilization of linear systems. Technical Report 05125, LAAS-CNRS, Toulouse, FRANCE, March 2005.

[4] H.S. Hussain and A.M. Annaswamy. Robust adaptive control in presence of unmodeled dynamics: a counter to rohrs's counterexample. In AIAA Guidance, Navigation, and Control (GNC) Conference, Boston, USA, 2013. 
[5] P. Ioannou and P. Kokotović. Adaptive Systems with Reduced Models. Springer-Verlag, Berlin, 1983.

[6] M. Krstic, I. Kanellakopoulos, and P. Kokotovic. Nonlinear and Adaptive Control Design. John Wiley and Sons, 1995.

[7] H. Leduc, D. Peaucelle, M. Lovera, and C. Pittet. Robust adaptive magnetic control of satellites with uncertain parameters. In IFAC World Congress, Toulouse, France, 2017.

[8] H. Leduc, D. Peaucelle, and C. Pittet. Adaptive control LMI-based design for descriptor systems rational in the uncertainties. In IFAC Workshop on Adaptation and Learning in Control and Signal Processing, pages 135-140, June 2016.

[9] H. Leduc, D. Peaucelle, and C. Pittet. LMIbased design of a robust direct attitude control for a satellite with uncertain parameters. In Automatic Control in Aerospace, pages 367-372, August 2016.

[10] D.P. Lindorff and R.L. Carroll. Survey on adaptive control using Liapunov design. Int. J. Control, 18(5):897-914, 1973.

[11] K. Lu and Y. Xia. Finite-time attitude stabilization for rigid spacecraft. International Journal of Robust and Nonlinear Control, 2013.

[12] A.R. Luzi, D. Peaucelle, J.-M. Biannic, C. Pittet, and J. Mignot. Structured adaptive attitude control of a satellite. Int. J. of Adaptive Control and Signal Processing, 28(7-8):664-685, 2014.

[13] R. Luzi. Commande variante dans le temps pour le contrôle d'attitude de satellites. $\mathrm{PhD}$ thesis, Université de Toulouse, February 2014.

[14] I. Mizumoto, H. Tanaka, and Z. Iwai. 2 dof adaptive pid control with a parallel feedforward compensator for nonlinear systems. In International conference on networking, sensing and control, Okayama, March 2009.
[15] D. Peaucelle, B. Andrievsky, V. Mahout, and A. Fradkov. Robust simple adaptive control with relaxed passivity and PID control of a helicopter benchmark. In IFAC World Congress, 2011. Paper in an invited session.

[16] D. Peaucelle and A.L. Fradkov. Robust adaptive $L_{2}$-gain control of polytopic MIMO LTI systems - LMI results. Systems \& Control Letters, $57(11): 881-887,2008$.

[17] C. Pittet and D. Arzelier. DEMETER: a benchmark for robust analysis and control of the attitude of flexible microsatellites. In IFAC Symposium on Robust Control Design, pages 661-666, Toulouse, France, 2006.

[18] C. Pittet, A.R. Luzi, D. Peaucelle, J.-M. Biannic, and J. Mignot. In flight results of adaptive attitude control law for a microsatellite. In ESA Conference on Guidance, Navigation and Control Systems, Porto, June 2014.

[19] C. Pittet, J. Mignot, and C. Fallet. LMI based multi-objective $H_{\infty}$ control of flexible microsatellites. In IEEE Conference on Decision and Control, Sydney, Australia, 1999.

[20] L. Praly. Adaptive regulation: Lyapunov design with a growth condition. International journal of adaptive control and signal processing, 6:329351, January 1992.

[21] D. Taylor, P. Kokotovic, and R. Marino. Adaptive regulation of nonlinear systems with unmodeled dynamics. IEEE Transactions on Automatic Control, pages 405-412, 1989.

[22] J.-F. Tregouët, D. Arzelier, D. Peaucelle, C. Pittet, and L. Zaccarian. Reaction wheels desaturation using magnetorquers and static input allocation. IEEE Transactions on Control Systems Technology, pages 525-539, 2014.

[23] B.J. Yang, T. Yucelen, A.J. Calise, and J.Y. Shin. An LMI-based stability analysis for adaptive controllers. In American Control Conference, pages 2593-2598, 2009. 
[24] A.M. Zanchettin, A. Calloni, and M. Lovera. Robust magnetic attitude control of satellites. IEEE Transactions on Mechatronics, pages 1259-1268, August 2013.

[25] Z. Zhu, Y. Xia, and M. Fu. Adaptive sliding mode control for attitude stabilization with actuator saturation. IEEE Transactions on Industrial Electronics, 58:4898-4907, 2011. 